\title{
O Som das Coisas: Promovendo o Engajamento em um Ambiente de Aprendizagem Criativa
}

\author{
Marcelo Bender Machado ${ }^{1,2}$, Eduardo Abreu Xavier ${ }^{1}$, Andréia Sias Rodrigues ${ }^{1,2}$, \\ Kelen Bernardi ${ }^{1}$, Tiago Primo ${ }^{1}$, Tatiana Aires Tavares ${ }^{1}$ \\ ${ }^{1}$ Programa de Pós-Graduação em Computação - Universidade Federal de pelotas (UFPel) \\ Caixa Postal 15.064 - 91.501-970 - Pelotas - RS - Brazil \\ ${ }^{2}$ Instituto Federal de Educação, Ciência e Tecnologia Sul-rio-grandense (IFSul) \\ \{mb.machado, eaxavier, andreia.sias, tiago.primo, tatiana\}@inf.ufpel.edu.br \\ kelenbernardi@gmail.com
}

\begin{abstract}
This paper describes the results of a workshop evaluation focused on creating tangible interfaces using creative learning as a pedagogical methodology. Makey Makey, a platform for creating tangible interfaces, associated with Scratch software, was used to teach basic music concepts. An evaluation was conducted with 16 students, observing their engagement and motivation during the activity. For this, the SAM - Self Assessment Manikin methodology was applied, complemented by two other self-report and observation instruments that indicated in the emotional aspects engagement and motivation, indicating the effectiveness of the proposals set and alignment with the methodology guidelines.
\end{abstract}

Resumo. O artigo descreve os resultados da avaliação de uma oficina voltada para a criação de interfaces tangíveis utilizando aprendizagem criativa como metodologia pedagógica. Foi usado o Makey Makey, uma plataforma para a criação de interfaces tangíveis, associada ao Scratch para ensinar conceitos básicos de música. Realizou-se uma avaliação com 16 alunos, observando o seu engajamento e a motivação durante a atividade. Para isso, foi aplicada a metodologia SAM - Self Assessment Manikin complementado por outros dois instrumentos auto-avaliativos e observação que indicaram uma resposta emocional e alinhamento com as diretrizes da metodologia apontando engajamento e motivação o que indica a efetividade do conjunto da proposta.

\section{Introdução}

Com o crescimento da população, um dos grandes desafios da sociedade atual é como passar para as novas gerações o conhecimento disponível de modo a não gerar soluções de continuidade no processo de desenvolvimento humano. Para tanto é necessário pensar sob 2 aspectos, um relacionado ao acesso ao manancial de conhecimento gerado e outro com relação à forma como ele é passado às gerações. Este último relaciona-se ao aprimoramento das metodologias e técnicas de forma a torná-las mais eficientes e eficazes no sentido do aprendizado deste conhecimento. Soluções a partir de perspectivas apenas instrucionais [Papert 1994] mostram-se obsoletas frente às novas necessidades humanas previstas para os próximos anos [Forum 2016]. No futuro, os trabalhadores precisarão 
VIII Congresso Brasileiro de Informática na Educação (CBIE 2019)

Anais do XXX Simpósio Brasileiro de Informática na Educação (SBIE 2019)

ser mais proativos, trabalhar em grupo e dar respostas criativas para problemas que nem existem ainda.

Nesta perspectiva, a abordagem dada pela Aprendizagem Criativa (AC) está em sintonia com estes desafios. Ela baseia-se em ações que aparecem de forma espiral e vão a cada ciclo agregando mais e mais valor ao conhecimento construído sem limitações externas, dependendo do engajamento ao processo em questão [Resnick 2007].

Atualmente, trabalhar com crianças do ensino fundamental é trabalhar com nativos digitais [Lemos 2009]. Em tese, espera-se que a tecnologia digital faça parte do seu cotidiano o que resulta na impossibilidade de se pensar em uma educação contextualizada com a vida dos estudantes sem se levar em conta o uso, aplicação e até uma possível mediação a partir de dispositivos tecnológicos. Segundo Jane David, consultora da Apple, "existe perigo real em se considerar a tecnologia a salvadora da educação. Mas a educação não sobreviverá sem a tecnologia" [Oppenheimer 1997]. Sendo assim resolveu-se juntar estes dois mundos, o tecnológico através da aplicação do software Scratch [Kindergarten 2007] juntamente com uma plataforma de criação de dispositivos tangíveis (TUI - Tangible User Interface) conhecida por Makey Makey [Collective and Shaw 2012] atuando em um ambiente de AC [Resnick 2007] para o ensino de conceitos básicos de música, de modo a potencializar a experiência de aprendizagem, na hipótese de que estes elementos associados refletiriam em maior engajamento à atividade proposta.

O desafio foi a elaboração de uma atividade educacional com propósito de criação de um protótipo de TUI em forma de instrumento musical. As oficinas foram aplicadas a alunos cursando as disciplinas finais do ensino fundamental no sistema formal brasileiro de educação.

Para tanto, ao fim da oficina foi feita uma avaliação com relação à resposta emocional dos participantes através do protocolo de auto-relato SAM (Self Assessment Manikin) complementado por outros dois instrumentos auto-avaliativos de modo a captar a percepção dos mesmos quanto às diretrizes da $\mathrm{AC}$ e do conhecimento prévio dos participantes com a metodologia. De modo complementar procedeu-se a avaliação por especialistas durante a tarefa o que auxiliou na identificação de práticas, métodos, pistas sobre a relevância da abordagem.

Este artigo está estruturado da seguinte forma: Na próxima seção são discutidos os principais conceitos teóricos relacionados ao tema. Na seção 3, são apresentados trabalhos correlatos. A metodologia aplicada no estudo é descrita na seção 4, e também são destacados aspectos construtivos das oficinas realizadas bem como detalhes de sua aplicação. Na seção 5, são apresentados e discutidos os resultados. Por fim, na seção 6, são apresentadas as conclusões e possibilidades futuras para a continuidade da pesquisa.

\section{Referencial Teórico}

Jean William Fritz Piaget, trabalhou em suas pesquisas na perspectiva de descobrir os processos de desenvolvimento da inteligência humana a partir da interação. Basicamente, na perspectiva da interação com "as coisas", Piaget aponta que a aquisição de conhecimentos depende das estruturas cognitivas do indivíduo e da interação dele com o seu meio.

Piaget defendia que as crianças, para construir sua base de conhecimento, deveriam ir das operações concretas para as operações formais [Piaget 2013], Papert, avança 
VIII Congresso Brasileiro de Informática na Educação (CBIE 2019)

Anais do XXX Simpósio Brasileiro de Informática na Educação (SBIE 2019)

na teoria construtivista de Piaget criando uma escola chamada construcionista. Ele acreditava que o raciocínio abstrato não deveria ser visto mais avançado do que as manipulações concretas. No cerne do construcionismo está o conceito de "aprender fazendo" [Papert and Harel 1991].

Dando prosseguimento ao pensamento de Papert, Mitchel Resnick, quatro décadas depois do lançamento da linguagem LOGO, lança a linguagem de programação SCRATCH, avançando a partir do construcionismo, e sua teoria ficou conhecida como Aprendizagem Criativa (AC) [Pascoal 2017].

O conceito de AC desenvolvido no "Lifelong Kindergarten" do Instituto de Tecnologias de Massachusetts (MIT) baseia-se em ações que aparecem de forma espiral e vão a cada ciclo agregando mais e mais valor ao conhecimento construído sem limitações externas, dependendo do engajamento ao processo em questão. Para [Resnick 2007], no jardim da infância as crianças "imaginam o que querem fazer, criam um projeto baseado nas suas próprias ideias, brincam com suas criações, compartilham suas ideias $e$ criações com os outros, refletem sobre as próprias experiências, e isso tudo as leva a imaginar novas ideias e novos projetos". Imaginar, criar, brincar, compartilhar e refletir são passos que se desenvolvem durante o processo; embora a criança nem sempre os siga sequencialmente, estão sempre presentes.

Para que o processo de AC ocorra, e a espiral aconteça, são necessário os quatro Ps da AC, são eles: Projetos - Atividades construídas a partir de projetos significativos; Parcerias - no compartilhamento de ideias e colaboração entre os integrantes da atividade; Paixão - se norteia por despertar o entusiasmo por aquilo que está executando e Aprender Brincando - quando a experiência envolve os pares e gera diversão [Resnick 2014]. Em síntese, a pessoa que está neste processo é levada a buscar, cognitivamente, uma solução, em seguida produzindo a solução, analisando os passos até então e melhorando-a a partir desta reflexão sobre o que foi produzido.

A partir desta premissa, é apresentado neste trabalho uma proposta de atividade de ensino com o propósito de manter um alto nível de engajamento e motivação nos alunos, de modo a potencializar a aprendizagem. Nesta perspectiva, a proposta foi avaliar o conjunto da obra quanto ao engajamento e motivação dos alunos à atividade, na hipótese destes aspectos representarem elementos potencializadores no processo de aprendizagem dos participantes.

\subsection{Trabalhos Correlatos}

Nesta sessão são apresentados três trabalhos que mantêm relação com o proposto no que tange a construção de TUIs pelos alunos assim potencializando a aprendizagem abrangendo não só competências técnicas, mas de modo concomitante e transversal, também competências socioemocionais.

Em [da Silva and Gil 2019] o processo de aprendizagem não se limita à interação com a TUI. Com uma forma de trabalho herdada do faça você mesmo - DIY - o participante cria sua TUI e após interage com ela, assim, aprende as competências diretas que estão no seu foco de seu desenvolvimento (princípios de eletrônica) aliado às que obteve durante o processo de criação e construção do dispositivo (programação do arduino, por exemplo). Aqui percebe-se o conceito do construcionismo de Papert ampliando as possibilidades de aprendizagem para outras nem tão explícitas como as socioemocionais. 
VIII Congresso Brasileiro de Informática na Educação (CBIE 2019)

Anais do XXX Simpósio Brasileiro de Informática na Educação (SBIE 2019)

Diferentes habilidades, hard e soft skills, foram abordadas no processo de aprendizagem ampliando as capacidades de um dispositivo de interação convencional.

Em [Mäkelä and Vellonen 2018] é feito um estudo no sentido de mapear as possibilidades de kits de bricolage serem usados como ferramentas para educação especial. Para tanto, o kit escolhido foi o makey makey. Foram usadas oficinas, questionários e entrevistas com profissionais envolvidos no uso deste dispositivo. Os resultados indicam que este método pode proporcionar aos educadores especiais um papel mais ativo e criativo na adoção de tecnologia e beneficiar a educação especial, aumentando as possibilidades de acessibilidade, motivação e interação.

Com objetivo de pesquisar métodos para ensino de computers skills é proposto em [Bers 2018] uma metodologia que utiliza o software Scratch Junior e o kit robótico $K I B O$ em crianças. Neste paper também há o casamento do software com hardware para a construção de algo tangível pelas crianças que, ao participar do processo criativo e lúdico com as ferramentas disponíveis, vão desenvolvendo não só as competências técnicas como também de ordem socioemocionais.

\section{Metodologia}

Para a realização desta pesquisa, foram elaboradas duas oficinas, com a abordagem pedagógica baseada em AC, onde foram selecionados 16 alunos matriculados nas séries finais do ensino fundamental.

Os objetivos pedagógicos utilizados foram extraídos das recomendações da Base Nacional Comum Curricular (BNCC) [Ministério da Educação 2017]. Numa análise geral, a oficina foi construída na perspectiva de que fossem oportunizadas aos participantes trabalharem com as 10 competências gerais da Educação Básica conforme o documento base, de uma forma ou outra, em maior ou menor nível de profundidade, uma vez que a metodologia implica no aluno traçar sua trajetória de aprendizagem de forma pessoal e livre. De forma específica, optou-se por dar ênfase às competências e habilidades relacionadas à componente curricular arte, linguagem Música, do bloco relativo às séries finais do ensino fundamental ( $6^{\circ}$ ao $9^{\circ}$ ano) de códigos EF69AR20, EF69AR21, EF69AR22, EF69AR23.

O processo de design instrucional da atividade proposta seguiu as seguintes etapas:

1. Introdução: Apresentação dos objetivos da atividade e conceitos envolvidos, contextualização através de exemplos, demonstração dos materiais e ferramentas (papelão, papel, grafite, lápis de cor, canudos coloridos, fios, garrafas e tampas recicláveis, fita adesiva, fio elétrico, palitos de bambu). Em seguida apresentação do Scratch e Makey Makey. (Tempo previsto de 30 minutos).

2. Mão na massa: Execução da atividade contendo as etapas de projeto, montagem e conexão do instrumento com a plataforma Makey Makey e Scratch bem como testes de funcionalidade. Reflexão seguida por nova etapa de refinamento do projeto criado. (Tempo previsto de 45 minutos).

3. Apresentação: Momento de apresentação por parte dos alunos do que foi por eles criado para os professores e colegas sempre seguido por perguntas e questionamentos que levem a novas reflexões. Em seguida é dado um tempo para a criação de um trecho musical com o projeto feito e posterior registro com linguagem própria não formal (tempo previsto de 15 minutos). 
VIII Congresso Brasileiro de Informática na Educação (CBIE 2019)

Anais do XXX Simpósio Brasileiro de Informática na Educação (SBIE 2019)

\subsection{Planejamento da Oficina}

Para auxiliar na adaptação da dinâmica da oficina e à familiarização com os procedimentos propostos através de uma experiência prévia, foi utilizada a técnica do grupo focal [Morgan 1997]. Esta abordagem nos permitiu entender melhor os instrumentos utilizados e os procedimentos propostos através da experiência prévia dos mesmos. A oficina foi aplicada para 7 adultos (4 homens e 3 mulheres), todos alunos de um programa de PósGraduação em Computação. Os participantes foram voluntários e não representavam o público alvo da oficina. Cabe salientar que nenhum dos participantes tinha conhecimento prévio formal de teoria musical.

Os integrantes foram divididos em dois grupos. Ao final da atividade, os grupos desenvolveram dois instrumentos e um dos grupos escreveu uma composição dos sons. Esta composição foi escrita a partir das posições da representação das conexões do Makey Makey utilizadas no instrumento criado. Após, passou-se à atividade do grupo focal. Foram feitas 11 perguntas pelo moderador no sentido de levantar as impressões sobre determinados pontos da oficina. Baseado nas respostas dos diálogos, elencaram-se cinco categorias: (1) aplicabilidade e execução da oficina, (2) materiais, (3) metodologia de avaliação, (4) pontos fortes e (5) pontos fracos.

Em síntese, para os participantes da atividade, a oficina atendeu às expectativas em sintonia com os objetivos iniciais, principalmente no que tange a eficiência do método, reunindo elementos altamente motivantes numa atmosfera que incentivasse o aprendizado reforçando o pensamento criativo. Foram levantados pontos para a melhoria da atividade, como: melhoria da parte introdutória, foco em exemplos, desconstruindo préconceitos formais, mudança nos objetivos, o tempo inadequado para se realizar todas as tarefas, escrita da música inclusive. Estes elementos foram levados em consideração para a aplicação da oficina com o público alvo.

\subsection{Aplicação da Oficina com o Público-Alvo}

A aplicação da oficina aconteceu com dois grupos distintos na cidade de Pelotas, estado do RS. No primeiro encontro reuniram-se cinco alunos ( 2 meninos e 3 meninas), três deles oriundos de escolas particulares. Eles já tinham familiaridade com atividades extraclasse que envolviam tecnologias da informação, robótica ou clubes de computação. Apenas dois deles frequentavam a mesma escola, mas nenhum frequentava a mesma sala de aula. O segundo grupo era composto por 11 alunos (8 meninos e 3 meninas), ambos da mesma escola pública municipal, mas não da mesma turma. $\mathrm{O}$ vínculo era que frequentavam o clube de robótica da escola no mesmo turno.

A oficina, com os dois grupos, foi conduzida em dois dias distintos e com apenas pequenas diferenças na etapa de introdução, sendo que para o segundo grupo limitouse, na explicação do funcionamento da plataforma Makey Makey e os efeitos de sua atuação, enquanto para o primeiro grupo estudado foram comentados, além deste aspecto, ainda princípios de eletricidade para compreensão do seu funcionamento. A duração das oficinas foi de 1 hora e 30 minutos. Ambas foram aplicadas no primeiro semestre de 2019.

Para avaliação da atividade pelos alunos, foram escolhidos dois tipos de processos avaliativos, observação e aplicação de questionários de auto-avaliação. 
VIII Congresso Brasileiro de Informática na Educação (CBIE 2019)

Anais do XXX Simpósio Brasileiro de Informática na Educação (SBIE 2019)

Para a auto-avaliação, foi escolhido o instrumento SAM - Self Assessment Manikin [Bradley and Lang 1994] que consiste em um questionário orientado por imagem, desenvolvido para avaliar as dimensões de satisfação, motivação e controle provenientes da realização de uma tarefa (no caso específico desta pesquisa). Foi escolhido por constituir em um método já estável e amplamente utilizado por pesquisas na área de interação humano-computador e por também conseguir representar uma medida breve (apenas três questões a serem respondidas) e não-verbal (pictórico) do estado emocional. [Bynion and Feldner 2017].

De modo a complementar a avaliação propôs-se uma alternativa pictórica como em [Koster et al. 2016] apresentado na Figura 1 onde se pretende colher a avaliação dos participantes quanto a se sentiram-se desafiados (Projetos), envolvidos (Paixão), colaborativos (Parcerias) e se divertiram-se (Aprender Brincando), ou seja, tentou-se captar a percepção dos participantes quanto aos pilares da metodologia de AC. Ainda solicitou-se aos participantes da atividade que respondessem de forma descritiva as perguntas: (1) Você gostaria de fazer outra oficina como esta? (2)Do que você mais gostou? (3)Do que você menos gostou? (4)Você já tem na sua escola aulas deste tipo?

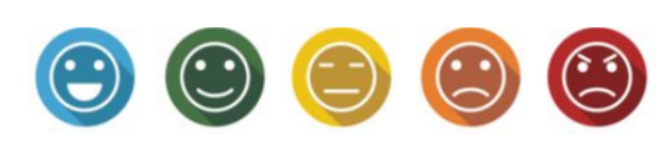

Figura 1. Os cinco estados emocionais usados no questionário para avaliação da percepção dos $4 \mathrm{Ps}$ da AC.

\section{Resultados e Discussão}

Na Figura 2 pode-se observar os instrumentos desenvolvidos pelos participantes.

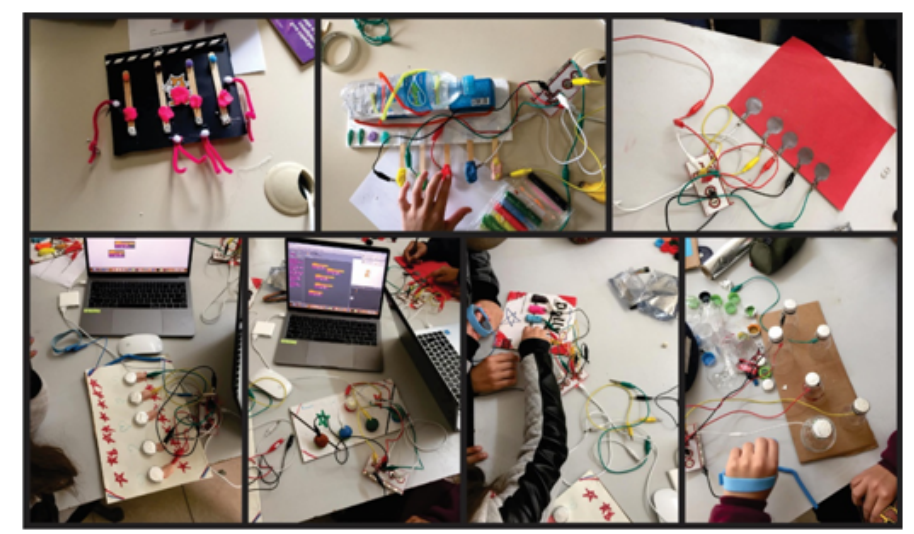

Figura 2. Instrumentos desenvolvidos nas oficinas. Ao todo foram 7 instrumentos desenvolvidos nas oficinas com o público-alvo.

Os resultados da aplicação do SAM, com os 16 participantes das oficinas, são sumarizados e ilustrados na Figura 3. A análise foi feita dividindo-se a escala de 9 pontos em 3 blocos. Os resultados situados no bloco de 1 a 3 foram considerados como não desejáveis indicando respectivamente desprazer (atividade foi desagradável), calma (atividade foi relaxante) e submissão (se manteve uma postura não ativa durante o processo). 
VIII Congresso Brasileiro de Informática na Educação (CBIE 2019)

Anais do XXX Simpósio Brasileiro de Informática na Educação (SBIE 2019)

Os resultados de 4 a 6 foram considerados como neutros. Presume-se que, ou o participante não quis se posicionar frente ao instrumento, ou não entendeu, ou não fez nenhuma relação de mérito para avaliar a atividade. Já os resultados de 7 a 9 foram considerados como desejáveis pois interpretou-se que os participantes se sentiram alegres e satisfeitos, exaltados e arrebatados e no controle do processo, respectivamente, ambas situações em maior ou menor grau.

A partir desta classificação, os resultados apontaram na direção da validação da pesquisa sendo que $81,25 \%$ das respostas indicaram que os participantes tiveram sentimentos de prazer e sentiram-se no controle das atividades enquanto $93,75 \%$ indicaram que ainda se sentiram excitados ou se mantiveram em estado de alerta durante a atividade.
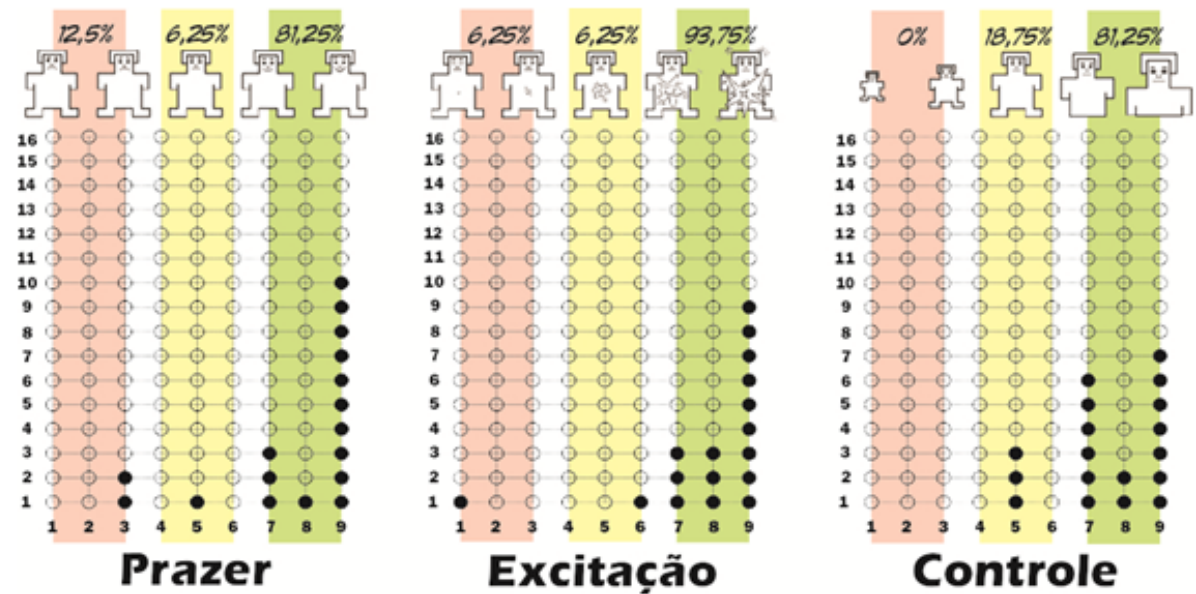

Figura 3. Resultado da avaliação do SAM.

Mesmo com resultados positivos a partir da análise de forma isolada das dimensões dadas pela aplicação do SAM, ainda se poderia ter resultados não esperados. Isto é avaliado a partir da análise que leva em conta a relação entre os resultados obtidos pela dimensão do prazer e da excitação. Na Figura 4(a) é apresentado o gráfico do espaço afetivo resultante [Lang and Bradley 2007]. No gráfico estão apresentadas 5 regiões $(\mathbf{a} ; \mathbf{b} ; \mathbf{c} ; \mathbf{d} ; \mathbf{e})$. A região com predominância de resultados foi a $\mathbf{b}$ com 13 pontos. Os resultados nesta região denotam que os estímulos recebidos durante a atividade foram sentidos de maneira mais prazerosa e excitante. Quanto mais a $45^{\circ}$ neste quadrante, pode dizer que o julgamento reflete uma "motivação apetitosa"enquanto que resultados no $4^{\circ}$ quadrante (área d) a $315^{\circ}$ no gráfico refletem uma motivação mais defensiva, ou seja, com estado de alerta porém com desprazer. Quando nenhum sistema motivacional está ativo o julgamento é neutro e a posição relacionada no gráfico é a área a. Neste estudo não foi encontrado nenhum resultado nesta área. Foram encontrados 1 resultado para cada uma das áreas c, e e que representam respectivamente calma/desprazer e estado de alerta, excitação e indiferença. Ambos estados indesejáveis como resultados para os objetivos da pesquisa.

O espaço afetivo dado pelas respostas do SAM, predominantemente ocupando o $1^{\circ}$ quadrante, revela uma resposta emocional positiva para a oficina, revelando que a mesma foi prazerosa e ao mesmo tempo excitante para os participantes, aspectos que se conectam de forma afirmativa às dimensões de motivação e engajamento corroborando com a potencialização do processo de aprendizagem pelos alunos. 

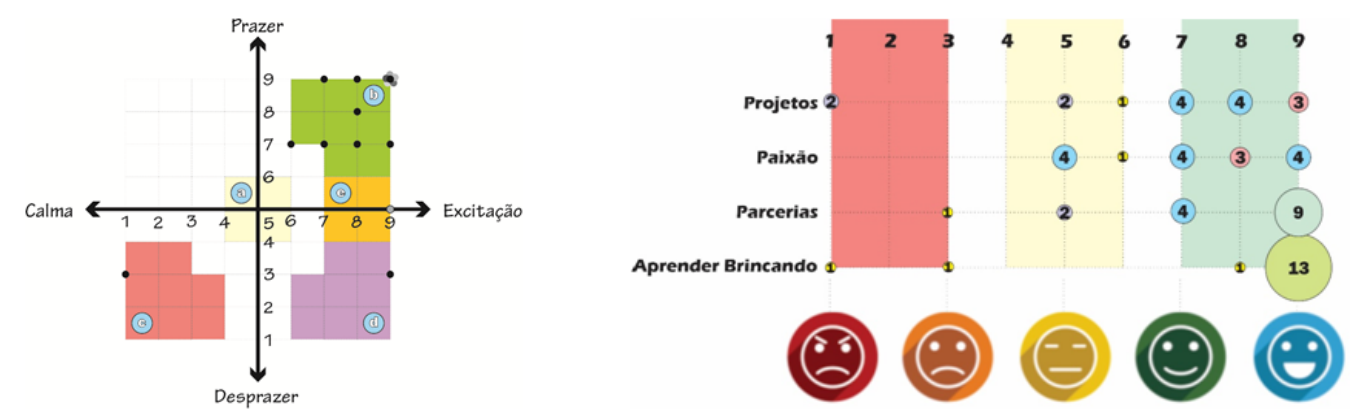

Figura 4. (a) Representação bidimensional do Espaço Afetivo encontrado a partir dos resultados da aplicação do SAM. (b) Resultado do questionário sobre a percepção dos participantes da oficina pelos "4 Ps" da AC. Fonte: Autor

Na Figura 4(b) têm-se os resultados do segundo questionário aplicado aos participantes da oficina. A expectativa com ela era que indicasse a aderência da oficina aos referenciais da AC a que são traduzidos pelos "4 Ps". O resultado é promissor para a questão do aspecto lúdico da experiência. Nela, pode-se auferir, que os participantes se sentiram à vontade, como que brincando. Também se percebe pelos resultados o reconhecimento das relações colaborativas que ocorreram durante o processo. Apesar de não ter tido muitos resultados máximos como nas dimensões anteriores, os resultados nos quesitos paixão e projetos também são satisfatórios porque, na soma dos últimos 3 pontos da escala, eles conseguem $68,75 \%$ das respostas.

Quanto aos resultados obtidos pelo questionário aplicado aos participantes da oficina, $100 \%$ das respostas foi que gostariam de ter mais experiências de ensino deste tipo e que não tinham tido ainda contato com esta metodologia. Quanto ao que mais gostaram, destacaram-se as respostas sobre "criar coisas novas", "aprender a fazer e tocar música", e da "invenção de cada grupo". Entre as respostas referentes ao que menos gostaram estavam que não tinham se desagradado com nada em primeiro lugar $(81,25 \%)$ e em segundo que queriam que a atividade durasse mais tempo $(18,75 \%)$.

Quanto aos resultados da observação da atividade realizou-se a seguinte análise crítica a partir de 5 pontos principais:

\section{Espiral Criativa:}

A percepção do processo de pensar e repensar dado pela espiral criativa foi observada nos dois grupos do mesmo modo, mesmo o primeiro se tratando de crianças na maioria oriundas de escolas particulares e com familiaridade com a linguagem Scratch, como com relação às crianças do segundo grupo, oriundas de escola pública e sem familiaridade com ferramentas de aprendizagem digitais.

Participação: Todos os participantes se mostraram muito envolvidos na atividade, desafiados e absorvidos pelo desafio proposto. A percepção de ter tido envolvimento até o último momento foi explícita. Tanto que após o término dos trabalhos foram questionados, informalmente, a respeito do tempo e muitos responderam que nem notaram que ela passou.

Teoria x Prática: A intenção de passar para os alunos a teoria formal do fenômeno envolvido na oficina mostrou-se menos efetiva do que quando surge a curiosi- 
dade e necessidade por parte do aluno, no meio do processo, com o fim de dar segmento à atividade ou por simples curiosidade de saber os fundamentos por trás do fenômeno. Este fato foi evidenciado entre as oficinas principalmente a partir da segunda, onde evitou-se, de modo proposital, na introdução ao funcionamento da platafroma Makey Makey, a fala sobre os princípios de eletricidade envolvidos no processo, o que acarretou que na hora da conexão, as respostas foram buscadas pelos alunos de forma natural.

Tempo: O tempo escolhido de uma hora e 30 minutos foi pouco para se exaurir as potencialidades de aprendizado a partir da espiral criativa. Percebeu-se isto porque ao fim do primeiro teste do instrumento, começavam a surgir indagações dos alunos do tipo como colocar mais notas musicais, as características elétricas de determinado elemento, de como melhorar o instrumento, de como deixá-lo mais fácil de tocar, o que remete a um processo mental onde a espiral da AC estava ativa.

Colaboração: Houve, dentro dos grupos forte conexão. Entre grupos ela não foi tão observada, mas foi observada em nível de colaboração de materiais e equipamentos. Para novas rodadas, de modo a estimular um comportamento mais colaborativo, deve-se criar momentos de parada, avaliação e feedback com os trabalhos dos outros grupos. Isto traria outra percepção para o trabalho colaborativo.

\section{Conclusões e Trabalhos Futuros}

O presente trabalho avaliou uma experiência em aprendizagem a partir de oficina aplicada a alunos do ensino fundamental, séries finais, baseada na metodologia de AC com o intuito de aprendizagem em conceitos básicos de música.

O conjunto dos resultados positivos em todos os instrumentos de avaliação indicam que a atividade gerou forte engajamento e motivação, fatores potencializadores no processo de aprendizagem. Estes resultados apontam caminhos para a continuidade do trabalho de pesquisa principalmente focada na validação da metodologia para aprendizagem em outras áreas do conhecimento bem como a viabilidade de design instrucional para aplicação da educação formal atendendo as diretrizes da BNCC Brasileira.

Em paralelo, os autores também entendem que para validações futuras sejam pesquisadas outras formas pictóricas de representação mais alinhadas com o cotidiano do público-alvo, pois apesar da facilidade dada pela metodologia de avaliação pictórica, os participantes tiveram de ser elucidados sobre como proceder na resposta aos questionários devido não perceberem de imediato o significado de alguns sentidos e imagens usadas para tal.

\section{Referências}

Bers, M. U. (2018). Coding, playgrounds and literacy in early childhood education: The development of kibo robotics and scratchjr. In 2018 IEEE Global Engineering Education Conference (EDUCON), pages 2094-2102. IEEE.

Bradley, M. M. and Lang, P. J. (1994). Measuring emotion: the self-assessment manikin and the semantic differential. Journal of behavior therapy and experimental psychiatry, 25(1):49-59.

Bynion, T.-M. and Feldner, M. (2017). Self-Assessment Manikin, pages 1-3. 
VIII Congresso Brasileiro de Informática na Educação (CBIE 2019)

Anais do XXX Simpósio Brasileiro de Informática na Educação (SBIE 2019)

Collective, B. M. and Shaw, D. (2012). Makey makey: improvising tangible and naturebased user interfaces. In Proceedings of the sixth international conference on tangible, embedded and embodied interaction, pages 367-370. ACM.

da Silva, C. S. G. and Gil, M. d. C. S. (2019). Creando material educativo: innovación, arduino y movimiento maker. Cuadernos de documentación multimedia, (30):129_ 144.

Forum, W. E. (2016). The future of jobs: Employment, skills and workforce strategy for the fourth industrial revolution. In Global Challenge Insight Report, World Economic Forum, Geneva.

Kindergarten, G. L. (2007). Scratch. https://scratch.mit.edu/credits. Acesso em 30/06/19.

Koster, A., Zilse, R., Primo, T., Oliveira, Á., Souza, M., Azevedo, D., Maciel, F., and Koch, F. (2016). Towards a digital teaching platform in brazil: findings from ux experiments. In International Conference on Learning and Collaboration Technologies, pages 685-694. Springer.

Lang, P. and Bradley, M. M. (2007). The international affective picture system (iaps) in the study of emotion and attention. Handbook of emotion elicitation and assessment, 29.

Lemos, S. (2009). Nativos digitais x aprendizagens: um desafio para a escola. Boletim Técnico do Senac, 35(3):38-47.

Mäkelä, S. and Vellonen, V. (2018). Designing for appropriation: A diy kit as an educator's tool in special education schools. International Journal of Human-Computer Studies, 118:14-23.

Ministério da Educação, B. (2017). Base nacional comum curricular. http: //basenacionalcomum.mec.gov.br/images/BNCC_EI_EF_110518_ versaofinal_site.pdf. Acesso em 4/07/19.

Morgan, D. L. (1997). Focus groups as qualitative research thousand oaks. Cal: Sage.

Oppenheimer, T. (1997). The computer delusion. The Atlantic Monthly, 280(1):45-62.

Papert, S. (1994). A máquina das crianças. Porto Alegre: Artmed.

Papert, S. and Harel, I. (1991). Situating constructionism. Constructionism, 36(2):1-11.

Pascoal, R. (2017). O que o mais importante instituto de tecnologia do mundo tem a ver com a sua aula. https://bit. Iy/2 Jtmc26. Acesso em 4/7/19.

Piaget, J. (2013). Principles of Genetic Epistemology: Selected Works vol 7. Routledge.

Resnick, M. (2007). All i really need to know (about creative thinking) i learned (by studying how children learn) in kindergarten. In Proceedings of the 6th ACM SIGCHI conference on Creativity \& cognition, pages 1-6. ACM.

Resnick, M. (2014). Give p'sa chance: Projects, peers, passion, play. In Constructionism and creativity: Proceedings of the Third International Constructionism Conference. Austrian Computer Society, Vienna, pages 13-20. 